\title{
Testicular tumors in children and adolescents: long-term endocrine and fertility issues
}

\author{
Katherine H. Anderson, Rodrigo L. P. Romao \\ Division of Pediatric Urology, IWK Health Centre, Department of Urology, Dalhousie University, Halifax, NS, Canada \\ Contributions: (I) Conception and design: All authors; (II) Administrative support: None; (III) Provision of study materials or patients: None; (IV) \\ Collection and assembly of data: All authors; (V) Data analysis and interpretation: All authors; (VI) Manuscript writing: All authors; (VII) Final \\ approval of manuscript: All authors. \\ Correspondence to: Dr. Rodrigo L. P. Romao. Division of Pediatric Urology, IWK Health Centre, 5850/5980 University Avenue, PO Box 9700, \\ Halifax, NS, B3K 6R8, Canada. Email: rodrigo.romao@dal.ca.
}

\begin{abstract}
The treatment modalities for testicular tumors (surgery, chemotherapy, and radiotherapy), have different associated gonadotoxic risks and the overall survival for most pediatric patients with testicular tumors is very good. However, necessary treatments may lead to the development of lasting gonadal dysfunction and subsequent negative health and quality of life impact. Research with long-term follow-up for patients who have undergone surgery as the sole treatment modality for testicular tumors in childhood are lacking. It is currently unclear if surgery leads to long-term negative functional outcomes. Alkylating agents (e.g., cyclophosphamide) have long been known to increase risk of infertility; platinumbased therapies used frequently for patients with germ-cell tumors (GCTs) also seem to carry some risk of gonadotoxicity, although they have not been as well studied. Radiotherapy to the gonads is toxic and Leydig cells are particularly sensitive to high doses of radiation (>12 Gy). Long-term fertility and hormonal impact vary based on the patient's age, as well as the type and intensity of the oncological treatment prescribed. Counselling regarding fertility risk and preservation options should ideally take place before initiating potentially gonadotoxic treatments. Hypogonadism in peri-pubertal boys can present as delayed onset or failure to progress through puberty. Sperm cryopreservation should be offered for post-pubertal boys who are able to provide a semen sample. For prepubertal boys or young males who cannot provide a semen sample, only experimental options are currently available. Much of the data reviewed here is extrapolated from research done on adult males whose reproductive and hormonal outcomes may not be comparable to younger patients who do not yet have fully developed reproductive systems. Currently, a lack of good quality evidence in this age range causes this restriction to be unavoidable. Patients and their families want to be informed of the risks and treatment options for preserving testicular function. As research continues in this field, it grows more important for urologists to be aware of the outcomes and options for their patients.
\end{abstract}

Keywords: Testicular tumors; children; adolescent; fertility; hypogonadism

Submitted Dec 27, 2019. Accepted for publication Apr 21, 2020.

doi: $10.21037 /$ tau-19-923

View this article at: http://dx.doi.org/10.21037/tau-19-923

\section{Background}

The incidence of testicular tumors in minors occurs in a bimodal pattern, with the first peak around age 2-3 years, and another in late teenage years. While testicular germ cell tumors (GCT) are among the most common solid tumors diagnosed in adolescents and young adults $(1,2)$, they are rare in the prepubertal population, comprising only $1 \%$ of all solid tumors in this group (3).

Akin to adult males, testicular tumors in pediatric patients often present as a painless mass (2). Ultrasonography is 
similarly useful in this population, and certain characteristic imaging findings can help to narrow the differential diagnosis, such as the classic "onion skin" appearance of an epidermoid cyst. The traditional biomarkers are used (AFP, beta-hCG, LDH), although some caveats exist. For example, infants have a physiologically elevated AFP which falls to normal adult male levels after 2 years of age; hence, interpretation of results requires following trends over time as well as utilizing available nomogram systems (4).

An important difference regarding testicular tumors in young males as opposed to adolescents/young adults is that a much higher proportion of benign tumors is seen in the prepubertal age group $(5,6)$. Single-centre studies identify mature teratoma and epidermoid cyst as the most common testicular tumors overall in young children (75\%) (7). In a recent review, yolk sac tumors (YST) were found to be the most common malignant subtype identified in boys less than 12 years of age (3).

Another significant point of distinction between children and adults is the occurrence of paratesticular rhabdomyosarcoma (RMS) in the pediatric and adolescent age group. Age of most common presentation for these tumors is also bimodal, with peaks in infancy (3-4 months of age) and adolescence (age 16) (8). While RMS is not a primary testicular malignancy, it typically arises from the spermatic cord and treatment does involve an orchiectomy and adjuvant chemotherapy with significant gonadotoxic potential.

Prognosis is generally favorable for GCT, mortality rate is estimated at only $3-6 \%$ in prepubertal boys and adolescents so it is paramount that clinicians remain cognizant of patients' post-treatment quality of life and reproductive function, since most of these patients will have lengthy life spans $(1,3)$. Paratesticular RMS prognosis is less favorable than GCT but still good with $80 \% 5$-year survival rates with current therapies (9).

In this review, we will focus on the long-term fertility and endocrine issues that can affect pediatric testicular cancer survivors. The associated gonadotoxicities of testicular cancer treatments and new therapies for fertility preservation will be highlighted. There may be important differences in endocrine/reproductive function impact when treatment takes place at a younger age during ongoing development. We recognize this as an unavoidable limitation due to the current paucity of available data in this age group.

\section{Mechanisms of gonadotoxicity in patients with testicular tumors}

\section{Surgery}

\section{Testicular sparing surgery}

Given the propensity for testicular tumors in prepubertal patients to be benign, testis-sparing surgery is favoured in many cases (7). Research has been conducted to ascertain if testis-sparing surgery is safe in this population (10). In one single-centre study, teratoma was found to be the most common testicular GCT; when testis-preserving surgery was done for patients with localized lesions, normal tumour markers, and sonographic findings suggestive of teratoma or epidermoid cyst, there were no tumor recurrences (10). These authors suggest that in prepubertal children with normal pre-operative serum AFP, testis-sparing surgery should be considered.

Similarly, at a multi-centre study, $74 \%$ of testicular tumors in prepubertal boys were found to be benign, so testis-sparing surgery was recommended (7). Another specific situation where partial orchiectomy may be favored would be in a case of a mass in a solitary testis. Testicularsparing surgery logically has the most minimal impact on long-term fertility and endocrine function, assuming this is the only treatment the patient requires.

\section{Radical surgery}

Unfortunately, not all testicular tumors will be amenable to testis-sparing surgery or harbor benign pathology. Whenever a malignant neoplasm of the testis is suspected, radical inguinal orchiectomy is indicated. To our knowledge, there are no studies that evaluate if there is increased risk of impaired spermatogenesis in children or adolescents who have undergone a unilateral orchiectomy as the sole treatment for testicular cancer (11). However, in adult men with testicular GCT, semen analysis abnormalities preorchiectomy are well established and these men may have higher rates of infertility (12).

Since pre-pubertal patients with testicular YST are usually treated with orchiectomy alone, this would be the ideal population to study the long-term impact of unilateral surgery on testicular function. Unfortunately, there are no reported long-term fertility or endocrine outcomes focused on this specific group to date (11).

Patients with malignant GCT who have metastatic nodal disease may require retroperitoneal lymph node dissection 
(RPLND). In RMS, a unilateral template is recommended for all patients older than 10 years of age, since the risk of occult metastases is $20-30 \%$ in this age group (13). Development of retrograde ejaculation following this surgery can occur, increasing the likelihood of fertility impairment. However, in a study of post-testicular cancer treatment fertility rates in adult men, $77 \%$ of men who had undergone RPLND were able to conceive without the use of assisted reproductive technologies (14).

Most patients with non-stage I GCT and RMS will receive multimodal therapy and therefore gonadotoxicity arises from a multiple hit mechanism.

\section{Chemotherapy}

Gonadotoxicity developing from exposure to certain chemotherapeutic drugs, such as alkylating agents (e.g., cyclophosphamide) used to treat leukemia and solid tumors like neuroblastoma and RMS, is well-established (15). In fact, models for prediction of the risk of infertility based on exposure to cyclophosphamide equivalent doses are available (16). However, the mainstay of GCT chemotherapies are platinum-based agents, and the effect of these on testicular function is uncertain, although reports of negative long-term fertility outcomes in men exposed to high doses of cisplatin have been published (14). In a recent clinical practice guideline, no studies were found for prepubertal boys that specifically studied cisplatin-based chemotherapy and effects on spermatogenesis (11).

Along with vincristine and actinomycin $\mathrm{D}$, cyclophosphamide is part of the backbone for RMS treatment regardless of location. Historically, patients with intermediate risk RMS received a total dose of $16.8 \mathrm{~g} / \mathrm{m}^{2}$, which is clearly in the gonadotoxic range with expected negative fertility outcomes. A recent Children's Oncology Group (COG) study showed that alternating vincristine and irinotecan with VAC cycles (known as VAC/VI) in intermediate risk RMS allowed for a reduction in the cyclophosphamide cumulative dose to $8.4 \mathrm{~g} / \mathrm{m}^{2}$ with preservation of good oncological outcomes (17).

\section{Radiotherapy}

Most research conducted on the effects of radiation treatment on testicular function has been done in other childhood cancers such as leukemia, lymphoma, and Wilms tumor (18). As seminoma is rarer in pre-pubertal boys, there is minimal research regarding effect of radiation post- radical orchiectomy in this population. Radiation to the testes in moderate to high doses can lead to permanent impaired fertility due to halted spermatogenesis (11). Data from the Childhood Cancer Survivor Study showed that radiation to the testes of $>7.5$ Gy decreased patients' rates of fathering a child as compared with their siblings who had not had childhood cancer (19).

Direct gonadal radiation exposure higher than 12 Gy and total body irradiation (TBI) are recognized as gonadotoxic (11). Although not a primary testicular tumor, it is important to be aware that the testis is considered a "sanctuary" site for acute lymphoblastic leukemia (ALL). ALL testicular relapses have historically been treated with high radiotherapy doses (24 Gy), which are well above the toxic range. In recent years, cooperative groups have made a concerted effort to reduce radiation exposure in this particular population through the use of systemic agents that cross the blood-testis barrier, such as methotrexate (20).

A summary of the mechanisms underlying gonadotoxicity from testicular tumor treatment can be seen in Table 1 .

\section{Impact of treatment for testicular tumors on long-term fertility outcomes}

Overall, fertility rates after treatment for testicular cancer are acceptable and related to the intensity of multimodal gonadotoxic treatment received. In a large cross-sectional study involving almost 1,500 adult testicular cancer survivors among whom 554 attempted post-treatment conception, fertility rates were as high as $92 \%$ (95\% CI: $78 \%$ to $98 \%$ ) for patients who underwent orchiectomy alone and as low as $48 \%$ (95\% CI: $30 \%$ to $69 \%$ ) for patients who received high-dose chemotherapy $(850 \mathrm{mg}$ of cisplatin). In this study, $22 \%$ of patients resorted to assisted reproductive technologies for conception. Dry ejaculation secondary to RPLND was an important negative predictor of post-treatment fatherhood on multivariable analysis (14).

The same Norwegian group went on to report that the overall 15 -year actuarial paternity rate was $85 \%$ in 106 men eligible for the paternity analyses who underwent surgery (orchiectomy +/- RPLND) and 2 to 4 cycles of chemotherapy; increased number of platinum-based chemotherapy cycles was associated with decreased paternity rates (21).

Currently, data on long-term reproductive outcomes for children treated for testicular tumors are not available. Nonetheless, the potential of fertility impairment through the mechanisms described previously is real and should be 
Table 1 Mechanisms of gonadotoxicity in pediatric patients with testicular tumors

\begin{tabular}{lll}
\hline Treatment modality & Fertility impact & Endocrine impact \\
\hline Surgery & Unlikely & Unlikely \\
Testis-sparing & Uncertain* & Uncertain* \\
Radical orchiectomy & Possible: retrograde ejaculation & None \\
RPLND & & Uncertain* \\
Chemotherapy & Possible in patients receiving high doses/multiple cycles of cisplatin & Possible: dose dependent \\
Platinum-based & (Broyd ref) & \\
Alkylating agents & Established and dose-dependent: more likely after exposure & \\
& $>4,000$ mg/m² of cyclophosphamide equivalents (Green ref.) & Established: gonadal exposure $>12$ Gy \\
\hline
\end{tabular}

*Uncertain: no studies or minimal studies with long-term follow-up. RPLND, retroperitoneal lymph node dissection.

kept in mind by healthcare providers before the initiation of treatment so a discussion about fertility preservation options can occur.

\section{Impact of treatment for testicular tumors on long-term endocrine outcomes}

Leydig cells are less sensitive to chemotherapy than Sertoli cells. Hence, exposed patients are more likely to depict impaired spermatogenesis with consequent infertility rather than testosterone deficiency and/or delayed onset of puberty. Conversely, radiation-induced Leydig cell failure is dose-dependent (22).

A few studies have suggested that childhood cancer survivors, including survivors of testicular cancer are at risk for hypogonadism $(15,23,24)$. Nonetheless, most of these reports are based on a biochemical definition [low levels of testosterone +/- high levels of luteinizing hormone (LH)] rather than a clinical one.

Isaksson et al. reported an odds ratio for biochemical hypogonadism of 2.1 (95\% CI: 1.1-4.1) in 121 childhood cancer survivors compared to controls; six of these patients were survivors of testicular cancer, three of which were hypogonadal based on the author's definition. The same authors reported an odds ratio of 2.3 (95\% CI: 1.1-4.7) for hypogonadism in 89 adult testicular cancer survivors $v s$. controls, with no substantial difference between seminoma and non-seminoma cases (15). They conclude that their results would support a policy for screening survivors of testicular cancer for androgen insufficiency.
In one of the few studies assessing clinical signs of hypogonadism, Lackner $e t a l$. identified androgen deficiency symptoms in 23/68 (33.8\%) adult testicular cancer survivors using a validated questionnaire. Interestingly, the authors also reported that no testosterone level threshold for development of symptoms could be established (25).

A recent study of the St Jude Lifetime Cohort of childhood cancer survivors $(n=1,516)$ found that both radiation therapy, exposure to a cyclophosphamide equivalent dose $>4,000 \mathrm{mg} / \mathrm{m}^{2}$ and unilateral orchiectomy $(n=35)$ were associated with Leydig cell failure (defined as low testosterone and elevated LH) and dysfunction (normal testosterone but elevated LH). Furthermore, survivors with Leydig cell failure were more likely to develop increased waist circumference, diabetes, erectile dysfunction and had increased all-cause mortality on multivariable analysis (26).

Researchers interested in this topic should be aware that performing accurate measurements of sex steroid hormones and gonadotropins is challenging, as there is significant variation based on the time of day for sample collection and serum concentrations of sex hormone-binding globulin (24).

For pre-pubertal boys, testosterone deficiency from testicular cancer treatments can present as delayed onset of puberty or absence of secondary sex characteristics (27). Monitoring growth (height) and pubertal development (Tanner stage including testicular volume) is recommended for boys exposed to gonadotoxic treatments; measuring serum testosterone in the morning is probably indicated in patients at higher risk (i.e., radiation exposure over $12 \mathrm{~Gy}$ 
Table 2 Recommendations for long-term monitoring and interventions for pediatric patients with testicular tumors

\begin{tabular}{llll}
\hline Type of dysfunction & Clinical presentation & Diagnostic tests & Potential interventions \\
\hline $\begin{array}{l}\text { Endocrine } \\
\text { (Leydig cells) }\end{array}$ & $\begin{array}{l}\text { Delayed onset or failed to progress through } \\
\text { puberty; clinical symptoms of hypogonadism } \\
\text { weight gain, sexual and erectile dysfunction }\end{array}$ & $\begin{array}{l}\text { Serum LH, testosterone } \\
\text { (morning sample) }\end{array}$ & Hormone replacement \\
$\begin{array}{l}\text { Reproductive } \\
\text { (Sertoli cells) }\end{array}$ & Infertility & Semen analysis & $\begin{array}{l}\text { Pre-treatment fertility preservation; } \\
\text { sperm banking; testicular } \\
\text { cryopreservation (experimental) }\end{array}$ \\
\hline
\end{tabular}

$\mathrm{LH}$, luteinizing hormone.

or TBI). Aside from recognition of hypogonadism symptoms, hormone replacement treatments in this age range are beyond the scope of the urologist. An appropriate time to refer a patient to a pediatric endocrinologist according to a clinical practice guideline is when a boy does not display signs of puberty by 14 years of age, has ceased to progress through puberty, and/or has a history of potentially gonadotoxic cancer treatments (11). See Table 2 for summary recommendations regarding monitoring and management of potential endocrine and reproductive abnormalities following testicular tumor management.

\section{Treatment for reduced fertility}

It is the standard of care to provide counselling about potential risks to fertility and options for preservation before any cancer treatment is initiated in all persons of reproductive age, including children (28). Failure to discuss the risks of decreased fertility from testicular cancer treatments and the potential preservation options can lead to future negative sentiment towards their care provider (29). This conversation will be varied based on the patient's age since fertility preservation options differ for pre- and postpubertal boys.

The gold standard for primary surveillance modality to evaluate spermatogenesis and fertility potential postgonadotoxic treatment is a semen analysis. The timing for investigation of fertility is up to the discretion of the patient after informed discussion has taken place as per expert opinion (11).

In adolescent boys who are able to provide a semen sample, established guidelines clearly recommend sperm cryopreservation prior to commencement of any cancer therapy as semen parameters may decrease post-surgery $(30,31)$. There are significant physical, social, emotional, financial, and mental barriers that may affect this specific age group which contributes to a low rate of sperm banking. Nonetheless, awareness and counselling about this topic by healthcare providers seem to be increasing (32).

In cases where post-pubertal patients are unable to produce a semen sample prior to surgery or other initiation of therapy, electroejaculation under general anesthesia is an option, although the upfront cost of the equipment is significant (32).

For the prepubertal age group, testicular biopsy and cryopreservation is offered by some centers $(33,34)$ but remains experimental at this point. The ability to generate viable pregnancies in rodents and primates from cryopreserved pre-pubertal testicular tissue offers encouragement that this option may become mainstream in the future $(35,36)$.

\section{Conclusions}

Multimodal therapy for testicular tumors in children and adolescents can have long-lasting negative reproductive and endocrine outcomes. Standard of care for young testicular cancer survivors should include prioritizing gonadal function both for maximizing healthy development and for quality of life benefits. Few studies have examined the longterm effect of cancer therapy on these outcomes in this age range.

Patients and their families expect to be informed regarding the potential risk to gonadal function, particularly the risk of infertility. Awareness about fertility counselling prior to initiation of any cancer therapy and fertility preservation options, even in the pre-pubertal age groups, is part of the knowledge urologists dealing with pediatric testicular tumors survivors are expected to master. Onset and progression through puberty constitute the most important endocrine milestones to be watched for in cancer survivors. 


\section{Acknowledgments}

None.

\section{Footnote}

Provenance and Peer Review: This article was commissioned by the Guest Editors (John Wiener, Jonathan Routh and Nicholas Cost) for the series "Pediatric Urologic Malignancies" published in Translational Andrology and Urology. The article was sent for external peer review organized by the Guest Editors and the editorial office.

Conflicts of Interest: All authors have completed the ICMJE uniform disclosure form (available at http://dx.doi. org/10.21037/tau-19-923). The series "Pediatric Urologic Malignancies" was commissioned by the editorial office without any funding or sponsorship. The authors have no other conflicts of interest to declare.

Ethical Statement: The authors are accountable for all aspects of the work in ensuring that questions related to the accuracy or integrity of any part of the work are appropriately investigated and resolved.

Open Access Statement: This is an Open Access article distributed in accordance with the Creative Commons Attribution-NonCommercial-NoDerivs 4.0 International License (CC BY-NC-ND 4.0), which permits the noncommercial replication and distribution of the article with the strict proviso that no changes or edits are made and the original work is properly cited (including links to both the formal publication through the relevant DOI and the license). See: https://creativecommons.org/licenses/by-nc-nd/4.0/.

\section{References}

1. Amini A, Waxweiler TV, Maroni PD, et al. Survival outcomes of adolescent and adult patients with nonseminomatous testicular germ-cell tumors: A populationbased study. J Pediatr Urol 2016;12:405.e1-405.e9.

2. Shaikh F, Murray MJ, Amatruda JF, et al. Paediatric extracranial germ-cell tumours. Lancet Oncol 2016;17:e149-62.

3. Maizlin II, Dellinger M, Gow KW, et al. Testicular tumors in prepubescent patients. J Pediatr Surg 2018;53:1748-52.

4. Blohm MEG, Vesterling-Hörner D, Calaminus G, et al. Alpha1-fetoprotein (AFP) reference values in infants up to
2 years of age. Pediatr Hematol Oncol 1998;15:135-42.

5. Taskinen S, Fagerholm R, Aronniemi J, et al. Testicular tumors in children and adolescents. J Pediatr Urol 2008;4:134-7.

6. Metcalfe PD, Farivar-Mohseni H, Farhat W, et al. Pediatric testicular tumors: contemporary incidence and efficacy of testicular preserving surgery. J Urol 2003;170:2412-5.

7. Pohl HG, Shukla AR, Metcalf PD, et al. Prepubertal testis tumors: Actual prevalence rate of histological types. J Urol 2004;172:2370-2.

8. Ahmed HU, Arya M, Muneer A, et al. Testicular and paratesticular tumours in the prepubertal population. Lancet Oncol 2010;11:476-83.

9. Hammond WJ, Farber BA, Price AP, et al. Paratesticular rhabdomyosarcoma: Importance of initial therapy. J Pediatr Surg 2017;52:304-8.

10. Shukla AR, Woodard C, Carr MC, et al. Experience with testis sparing surgery for testicular teratoma. J Urol 2004;171:161-3.

11. Skinner R, Mulder RL, Kremer LC, et al.

Recommendations for gonadotoxicity surveillance in male childhood, adolescent, and young adult cancer survivors: a report from the International Late Effects of Childhood Cancer Guideline Harmonization Group in collaboration with the PanCareSurFup Consort. Lancet Oncol 2017;18:e75-90.

12. Djaladat H, Burner E, Parikh PM, et al. The association between testis cancer and semen abnormalities before orchiectomy: A systematic review. J Adolesc Young Adult Oncol 2014;3:153-9.

13. Hamilton EC, Miller CC, Joseph M, et al. Retroperitoneal lymph node staging in paratesticular rhabdomyosarcomaare we meeting expectations? J Surg Res 2018;224:44-9.

14. Brydøy M, Fosså SD, Klepp O, et al. Paternity following treatment for testicular cancer. J Natl Cancer Inst 2005;97:1580-8.

15. Isaksson S, Bogefors K, Ståhl O, et al. High risk of hypogonadism in young male cancer survivors. Clin Endocrinol (Oxf) 2018;88:432-41.

16. Green DM, Liu W, Kutteh WH, et al. Cumulative alkylating agent exposure and semen parameters in adult survivors of childhood cancer: a report from the St Jude Lifetime Cohort Study. Lancet Oncol 2014;15:1215-23.

17. Hawkins DS, Chi YY, Anderson JR, et al. Addition of Vincristine and Irinotecan to Vincristine, Dactinomycin, and Cyclophosphamide does not improve outcome for intermediate-risk rhabdomyosarcoma: A report from the 
Children's Oncology Group. J Clin Oncol 2018;36:2770-7.

18. Wasilewski-Masker K, Seidel KD, Leisenring W, et al. Male infertility in long-term survivors of pediatric cancer: a report from the childhood cancer survivor study. J Cancer Surviv 2014;8:437-47.

19. Green DM, Kawashima T, Stovall M, et al. Fertility of male survivors of childhood cancer: a report from the Childhood Cancer Survivor Study. J Clin Oncol 2010;28:332-9.

20. Barredo JC, Hastings C, Lu X, et al. Isolated late testicular relapse of B-cell acute lymphoblastic leukemia treated with intensive systemic chemotherapy and response-based testicular radiation: A Children's Oncology Group study. Pediatr Blood Cancer 2018;65:e26928.

21. Brydøy M, Fosså SD, Klepp O, et al. Paternity and testicular function among testicular cancer survivors treated with two to four cycles of cisplatin-based chemotherapy. Eur Urol 2010;58:134-40.

22. Rose SR, Horne VE, Howell J, et al. Late endocrine effects of childhood cancer. Nat Rev Endocrinol 2016;12:319-36.

23. Romerius P, Ståhl O, Moëll C, et al. Hypogonadism Risk in Men Treated for Childhood Cancer. J Clin Endocrinol Metab 2009;94:4180-6.

24. Rogol AD, Anawalt BD. Should survivors of childhood cancer or testicular cancer be screened for androgen deficiency? Clin Endocrinol (Oxf) 2018;89:397-8.

25. Lackner JE, Märk I, Schatzl G, et al. Hypogonadism and androgen deficiency symptoms in testicular cancer survivors. Urology 2007;69:754-8.

26. Chemaitilly W, Liu Q, van Iersel L, et al. Leydig cell function in male survivors of childhood cancer: A report from the St Jude Lifetime Cohort Study. J Clin Oncol 2019;37:3018-31.

27. Viswanathan V, Eugster EA. Etiology and treatment of

Cite this article as: Anderson KH, Romao RLP. Testicular tumors in children and adolescents: long-term endocrine and fertility issues. Transl Androl Urol 2020;9(5):2393-2399. doi:10.21037/tau-19-923 hypogonadism in adolescents. Pediatr Clin North Am 2011;58:1181-200, x.

28. Oktay K, Harvey BE, Partridge AH, et al. Fertility preservation in patients with cancer: ASCO clinical practice guideline update. J Clin Oncol 2018;36:1994-2001.

29. Gupta AA, Donen RM, Sung L, et al. Testicular biopsy for fertility preservation in prepubertal boys with Cancer: Identifying Preferences for Procedure and Reactions to Disclosure Practices. J Urol 2016;196:219-24.

30. Anderson RA, Mitchell RT, Kelsey TW, et al. Cancer treatment and gonadal function: experimental and established strategies for fertility preservation in children and young adults. Lancet Diabetes Endocrinol 2015;3:556-67.

31. Liguori G, Trombetta C, Bucci S, et al. Semen quality before and after orchiectomy in men with testicular cancer. Arch Ital Urol Androl 2008;80:99-102.

32. Romao RLP, Lorenzo AJ. Fertility preservation options for children and adolescents with cancer. Can Urol Assoc J 2017;11:S97-102.

33. Ming JM, Chua ME, Lopes RI, et al. Cryopreservation of testicular tissue in pre-pubertal and adolescent boys at risk for infertility: A low risk procedure. J Pediatr Urol 2018;14:274.e1-5.

34. Corkum KS, Lautz TB, Johnson EK, et al. Testicular wedge biopsy for fertility preservation in children at significant risk for azoospermia after gonadotoxic therapy. J Pediatr Surg 2019;54:1901-5.

35. Yokonishi T, Ogawa T. Cryopreservation of testis tissues and in vitro spermatogenesis. Reprod Med Biol 2016;15:21-8.

36. Fayomi AP, Peters K, Sukhwani M, et al. Autologous grafting of cryopreserved prepubertal rhesus testis produces sperm and offspring. Science 2019;363:1314-9. 